\title{
Hyperbolic-parabolic singular perturbation for nondegenerate Kirchhoff equations with critical weak dissipation
}

\author{
Marina Ghisi \\ Università degli Studi di Pisa \\ Dipartimento di Matematica "Leonida Tonelli" \\ PISA (Italy) \\ e-mail: ghisi@dm.unipi.it \\ Massimo Gobbino \\ Università degli Studi di Pisa \\ Dipartimento di Matematica Applicata "Ulisse Dini" \\ PISA (Italy) \\ e-mail: m.gobbino@dma.unipi.it
}




\begin{abstract}
We consider the hyperbolic-parabolic singular perturbation problem for a nondegenerate quasilinear equation of Kirchhoff type with weak dissipation. This means that the dissipative term is multiplied by a coefficient $b(t)$ which tends to 0 as $t \rightarrow+\infty$.

The case where $b(t) \sim(1+t)^{-p}$ with $p<1$ has recently been considered. The result is that the hyperbolic problem has a unique global solution, and the difference between solutions of the hyperbolic problem and the corresponding solutions of the parabolic problem converges to zero both as $t \rightarrow+\infty$ and as $\varepsilon \rightarrow 0^{+}$.

In this paper we show that these results cannot be true for $p>1$, but they remain true in the critical case $p=1$.
\end{abstract}

Mathematics Subject Classification 2000 (MSC2000): 35B25, 35B40, 35L70.

Key words: singular perturbation, Kirchhoff equations, quasilinear hyperbolic equation, weak dissipation, energy estimates. 


\section{Introduction}

Let $H$ be a real Hilbert space. For every $x$ and $y$ in $H,|x|$ denotes the norm of $x$, and $\langle x, y\rangle$ denotes the scalar product of $x$ and $y$. Let $A$ be a self-adjoint linear operator on $H$ with dense domain $D(A)$. We assume that $A$ is nonnegative, namely $\langle A x, x\rangle \geq 0$ for every $x \in D(A)$, so that for every $\alpha \geq 0$ the power $A^{\alpha} x$ is defined provided that $x$ lies in a suitable domain $D\left(A^{\alpha}\right)$.

Let $m:[0,+\infty) \rightarrow(0,+\infty)$ be a function of class $C^{1}$ satisfying the nondegeneracy condition

$$
\mu_{1}:=\inf _{\sigma \geq 0} m(\sigma)>0 .
$$

We consider the second order Cauchy problem

$$
\begin{gathered}
\varepsilon u_{\varepsilon}^{\prime \prime}(t)+b(t) u_{\varepsilon}^{\prime}(t)+m\left(\left|A^{1 / 2} u_{\varepsilon}(t)\right|^{2}\right) A u_{\varepsilon}(t)=0 \quad \forall t \geq 0, \\
u_{\varepsilon}(0)=u_{0}, \quad u_{\varepsilon}^{\prime}(0)=u_{1},
\end{gathered}
$$

where $\varepsilon>0$ is a parameter and $b:[0,+\infty) \rightarrow(0,+\infty)$ is a given function. We also consider the first order reduced Cauchy problem

$$
\begin{gathered}
b(t) u^{\prime}(t)+m\left(\left|A^{1 / 2} u(t)\right|^{2}\right) A u(t)=0 \quad \forall t \geq 0, \\
u(0)=u_{0},
\end{gathered}
$$

obtained setting formally $\varepsilon=0$ in (1.2), and omitting the second initial condition in (1.3).

It is well known that (1.2), (1.3) is the abstract setting of a nonlocal quasilinear hyperbolic partial differential equation which was proposed as a model for the damped small vibrations of an elastic string or membrane with uniform density equal to $\varepsilon$. In the concrete setting assumption (1.1) is equivalent to strict hyperbolicity.

Following the approach introduced by J. L. Lions [12] in the linear case, we consider also the corrector $\theta_{\varepsilon}(t)$ as the solution of the second order linear problem

$$
\begin{gathered}
\varepsilon \theta_{\varepsilon}^{\prime \prime}(t)+b(t) \theta_{\varepsilon}^{\prime}(t)=0 \quad \forall t \geq 0, \\
\theta_{\varepsilon}(0)=0, \quad \theta_{\varepsilon}^{\prime}(0)=u_{1}+\frac{1}{b(0)} m\left(\left|A^{1 / 2} u_{0}\right|^{2}\right) A u_{0}=: w_{0} .
\end{gathered}
$$

Since $\theta_{\varepsilon}^{\prime}(0)=u_{\varepsilon}^{\prime}(0)-u^{\prime}(0)$, this corrector keeps into account the boundary layer due to the loss of one initial condition. Finally we define $r_{\varepsilon}(t)$ and $\rho_{\varepsilon}(t)$ in such a way that

$$
u_{\varepsilon}(t)=u(t)+\theta_{\varepsilon}(t)+r_{\varepsilon}(t)=u(t)+\rho_{\varepsilon}(t) \quad \forall t \geq 0 .
$$

The singular perturbation problem consists in proving that $r_{\varepsilon}(t) \rightarrow 0$ or $\rho_{\varepsilon}(t) \rightarrow 0$ in some sense as $\varepsilon \rightarrow 0^{+}$. 
Constant dissipation In the case of Kirchhoff equations this problem is well studied when $b(t)$ is a positive constant. The classical result (see [2], [16]) is the existence of a unique global solution provided that $\left(u_{0}, u_{1}\right) \in D(A) \times D\left(A^{1 / 2}\right)$ and $\varepsilon$ is small enough. Existence of a global solution with $\varepsilon$ large is still an open problem, as well as the nondissipative case $b(t) \equiv 0$.

The behavior of solutions as $t \rightarrow+\infty$ and $\varepsilon \rightarrow 0^{+}$has long been studied (see [4], [10], [7], [8]). A complete answer was found by H. НAshimoto and T. Yamazaki [11]. They proved that for initial data $\left(u_{0}, u_{1}\right) \in D\left(A^{3 / 2}\right) \times D(A)$ one has that

$$
\left|\rho_{\varepsilon}(t)\right|+(1+t)^{1 / 2}\left|A^{1 / 2} \rho_{\varepsilon}(t)\right|+\sqrt{\varepsilon}(1+t)\left|r_{\varepsilon}^{\prime}(t)\right| \leq C \varepsilon \quad \forall t \geq 0,
$$

where of course $C$ doesn't depend on $t$ and $\varepsilon$. When $\left(u_{0}, u_{1}\right) \in D\left(A^{2}\right) \times D(A)$ the coefficient $\sqrt{\varepsilon}$ may be dropped, thus providing a better convergence rate on $r_{\varepsilon}^{\prime}(t)$. We define (1.9) an error-decay estimate because it keeps into account in the same time both the decay of solutions as $t \rightarrow+\infty$, and their behavior as $\varepsilon \rightarrow 0^{+}$. It unifies and extends or improves all previous convergence or decay results.

This theory can be easily extended from the model case where $b(t)$ is constant to the more general case where $b(t)$ is bounded by two positive constants, with some extra conditions on $b^{\prime}(t)$.

Subcritical weak dissipation The case where $b(t) \rightarrow 0$ as $t \rightarrow+\infty$, the typical example being $b(t)=(1+t)^{-p}$ for some $p>0$, seems to be more difficult because of the competition between the smallness of $\varepsilon$ and the smallness of $b(t)$. To our knowledge the first results in this direction were obtained by K. ONO [14], who proved global existence in the special degenerate case $m(\sigma)=\sigma$ provided that $p \leq 1 / 3$, and by M. NAKAO and J. BAE [13] who proved global existence in the nondegenerate case with a coercive operator and a special nonlinear dissipation term which decays as $(1+t)^{-p}$ with $p<1$ as $t \rightarrow+\infty$.

Recently, T. YAMAZAKI [18] considered problem (1.2), (1.3) with the nondegeneracy assumption (1.1) and $b(t)$ which decays as $(1+t)^{-p}$ with $p<1$ as $t \rightarrow+\infty$. She proved existence of a global solution when $\varepsilon$ is small enough, and error-decay estimates for the singular perturbation problem.

The main result as stated in [18] depends on several parameters. Limiting for simplicity to estimates such as (1.9), she proved that for initial data $\left(u_{0}, u_{1}\right) \in D\left(A^{5 / 2}\right) \times D\left(A^{2}\right)$ one has that

$$
\left|\rho_{\varepsilon}(t)\right|+(1+t)^{(p+1) / 2}\left|A^{1 / 2} \rho_{\varepsilon}(t)\right|+\sqrt{\varepsilon}(1+t)\left|r_{\varepsilon}^{\prime}(t)\right| \leq C \varepsilon \quad \forall t \geq 0,
$$

where of course $C$ doesn't depend on $t$ and $\varepsilon$. The coefficient $\sqrt{\varepsilon}$ may be dropped for more regular data. This error-decay estimate extends (1.9) (which becomes the special case $p=0$ ) but for the fact that it seems to require more regularity on the initial data. 
In any case the time-decay rates on $\left|A^{1 / 2} \rho_{\varepsilon}(t)\right|$ and $\left|r_{\varepsilon}^{\prime}(t)\right|$ are those expected for solutions of the parabolic problem (1.4), (1.5), and they are optimal in the case of noncoercive operators in the sense explained in [3]. Roughly speaking this means that for $p<1$ the smallness of $\varepsilon$ is dominant over the smallness of $b(t)$ as $t \rightarrow+\infty$, and in this regime (1.2) behaves like a parabolic equation.

Proofs are based on a subtle spectral analysis of the corresponding linearized equations which gives at the same time the existence result and the decay-error estimates.

Supercritical and critical weak dissipation In this paper we consider problem (1.2), (1.3) in the case where $b(t)=(1+t)^{-p}$ with $p \geq 1$.

When $p>1$ a simple argument (see Theorem 2.3) shows that solutions of the hyperbolic problem (provided they globally exist, which remains an open problem) do not decay to 0 as $t \rightarrow+\infty$. On the contrary it is easy to see that the solutions of the parabolic problem always decay to 0 as $t \rightarrow+\infty$ (faster and faster as $p$ grows). As a consequence one cannot expect global-in-time error-decay estimates such as (1.10). This also means that for $p>1$ the smallness of $b(t)$ is dominant over the smallness of $\varepsilon$, and in this regime (1.2) behaves like a (nondissipative) hyperbolic equation.

This dichotomy, namely parabolic behavior for $p<1$ and hyperbolic behavior for $p>1$, had already been observed in [17] and [15] in the case of linear equations (when $m(\sigma)$ is a positive constant).

Then we concentrate on the critical case $p=1$, showing that also in this case the parabolic nature prevails. We prove indeed that for every $\left(u_{0}, u_{1}\right) \in D(A) \times D\left(A^{1 / 2}\right)$ the problem has a unique global solution provided that $\varepsilon$ is small enough, and this solution decays to 0 as $t \rightarrow+\infty$ with the same rate of the corresponding parabolic problem. We also prove decay-error estimates for the singular perturbation problem which extend (1.10) to the case $p=1$.

Our approach is based uniquely on energy estimates and it applies directly to the nonlinear problem. The main advantage is of course the possibility to treat the critical case. Nevertheless we point out that our assumptions on initial data are always minimal. We obtain indeed the global existence result for initial data in $D(A) \times D\left(A^{1 / 2}\right)$, which is of course the largest space where a solution of class $C^{2}$ can be expected, and we obtain the decay-error estimates for initial data in $D\left(A^{3 / 2}\right) \times D\left(A^{1 / 2}\right)$, which is the largest space where error estimates of order $\varepsilon$ can be obtained, even when $b(t)$ and $m(\sigma)$ are positive constants (see $[6])$. We also obtain the decay-error estimate without the coefficient $\sqrt{\varepsilon}$ for initial data $\left(u_{0}, u_{1}\right) \in D\left(A^{2}\right) \times D(A)$ (see Remark 2.4).

For the sake of simplicity we work out the details only in the case $p=1$. On the other hand, the same technique applies to the case $0<p<1$, or when $b(t)$ is constant, providing a different proof of (1.9) and (1.10) with minimal assumptions on the initial data.

When this paper was almost complete we were informed that Taeko Yamazaki inde- 
pendently obtained some results on the same problem (see [19]) by different methods.

\section{Statements}

Throughout this paper the operator $A$ always satisfies the following assumption:

$(\mathrm{Hp}-A) A$ is a selfadjoint nonnegative linear operator whose domain $D(A)$ is dense in a Hilbert space $H$.

We never assume $A$ to be coercive.

Existence of a unique global solution to problem (1.4), (1.5) is well known. The following is the result we need in this paper.

Theorem A Let $A$ be an operator satisfying (Hp-A), let $m:[0,+\infty) \rightarrow(0,+\infty)$ be a function of class $C^{1}$, let $b(t)=(1+t)^{-1}$, and let $u_{0} \in D(A)$.

Then problem (1.4), (1.5) has a unique solution

$$
u \in C^{1}([0,+\infty) ; H) \cap C^{0}([0,+\infty) ; D(A)) .
$$

Moreover $u \in C^{2}\left((0,+\infty) ; D\left(A^{\alpha}\right)\right)$ for every $\alpha \geq 0$.

If in addition $m$ satisfies (1.1), and $u_{0} \in D\left(A^{k / 2}\right)$ for some positive integer $k$, then there exists a constant $\gamma_{k}$, depending only on $k$ and $\mu_{1}$, such that

$$
\begin{gathered}
(1+t)^{2 k}\left|A^{k / 2} u(t)\right|^{2} \leq \gamma_{k}\left(\left|u_{0}\right|^{2}+\left|A^{k / 2} u_{0}\right|^{2}\right) \quad \forall t \geq 0, \\
\int_{0}^{+\infty}(1+s)^{2 k+1}\left|A^{(k+1) / 2} u(s)\right|^{2} d s \leq \gamma_{k}\left(\left|u_{0}\right|^{2}+\left|A^{k / 2} u_{0}\right|^{2}\right) .
\end{gathered}
$$

The existence part of Theorem $\mathrm{A}$ is an easy consequence of Theorem 4.1 in [9]. In section 3.2 below we sketch the proof of the decay estimates (2.1) and (2.2).

The first result of this paper concerns the global solvability of problem (1.2), (1.3), and the time decay of its solutions.

Theorem 2.1 Let $A$ be an operator satisfying (Hp-A), let $m:[0,+\infty) \rightarrow(0,+\infty)$ be a function of class $C^{1}$ satisfying the non-degeneracy condition (1.1), let $b(t)=(1+t)^{-1}$, and let $\left(u_{0}, u_{1}\right) \in D(A) \times D\left(A^{1 / 2}\right)$.

Then there exists $\varepsilon_{0}>0$ such that for every $\varepsilon \in\left(0, \varepsilon_{0}\right)$ problem (1.2), (1.3) has a unique global solution

$$
u_{\varepsilon} \in C^{2}([0,+\infty) ; H) \cap C^{1}\left([0,+\infty) ; D\left(A^{1 / 2}\right)\right) \cap C^{0}([0,+\infty) ; D(A)) .
$$

Moreover there exists a constant $C$, independent on $\varepsilon$ and $t$, such that

$$
\left|u_{\varepsilon}(t)\right|^{2} \leq C \quad \forall t \geq 0
$$




$$
\begin{gathered}
\left|A^{1 / 2} u_{\varepsilon}(t)\right|^{2} \leq \frac{C}{(1+t)^{2}} \quad \forall t \geq 0 ; \\
\left|u_{\varepsilon}^{\prime}(t)\right|^{2} \leq \frac{C}{(1+t)^{2}} \quad \forall t \geq 0 ; \\
\int_{0}^{+\infty}(1+s)\left(\left|u_{\varepsilon}^{\prime}(s)\right|^{2}+\left|A^{1 / 2} u_{\varepsilon}(s)\right|^{2}\right) d s \leq C ; \\
\varepsilon\left|A^{1 / 2} u_{\varepsilon}^{\prime}(t)\right|^{2}+\left|A u_{\varepsilon}(t)\right|^{2} \leq \frac{C}{(1+t)^{4}} \quad \forall t \geq 0 \\
\int_{0}^{+\infty}(1+s)^{3}\left(\left|A^{1 / 2} u_{\varepsilon}^{\prime}(s)\right|^{2}+\left|A u_{\varepsilon}(s)\right|^{2}\right) d s \leq C .
\end{gathered}
$$

The second result of this paper are the following decay-error estimates.

Theorem 2.2 Let $A, m, b$ be as in Theorem 2.1. Let $\left(u_{0}, u_{1}\right) \in D\left(A^{3 / 2}\right) \times D\left(A^{1 / 2}\right)$. Let $u_{\varepsilon}(t)$ be the solution of (1.2), (1.3), let $u(t)$ be the solution of (1.4), (1.5), and let $r_{\varepsilon}(t)$ and $\rho_{\varepsilon}(t)$ be defined by (1.8).

Then there exist $\varepsilon_{0}>0$ and $C>0$ such that for every $\varepsilon \in\left(0, \varepsilon_{0}\right)$ we have that

$$
\begin{gathered}
\left|\rho_{\varepsilon}(t)\right| \leq C \varepsilon \quad \forall t \geq 0 ; \\
\left|A^{1 / 2} \rho_{\varepsilon}(t)\right| \leq C \frac{\varepsilon}{1+t} \quad \forall t \geq 0 ; \\
\left|r_{\varepsilon}^{\prime}(t)\right| \leq C \frac{\sqrt{\varepsilon}}{1+t} \quad \forall t \geq 0 ; \\
\int_{0}^{+\infty}(1+s)\left|A^{1 / 2} \rho_{\varepsilon}(s)\right|^{2} d s \leq C \varepsilon^{2} ; \\
\int_{0}^{+\infty}(1+s)\left|r_{\varepsilon}^{\prime}(s)\right|^{2} d s \leq C \varepsilon^{2} .
\end{gathered}
$$

The last result of this paper is that in the supercritical case $p>1$ solutions cannot decay to 0 as $t \rightarrow+\infty$.

Theorem 2.3 Let $A$ and $m$ be as in Theorem 2.1. Let $b:[0,+\infty) \rightarrow(0,+\infty)$ be a continuous function such that

$$
\int_{0}^{+\infty} b(s) d s<+\infty
$$

Let $\left(u_{0}, u_{1}\right) \in D(A) \times D\left(A^{1 / 2}\right)$ be such $\left|u_{1}\right|^{2}+\left|A^{1 / 2} u_{0}\right|^{2}>0$. Let us assume that for some $\varepsilon>0$ problem (1.2), (1.3) has a global solution $u_{\varepsilon}$.

Then

$$
\liminf _{t \rightarrow+\infty}\left(\left|u_{\varepsilon}^{\prime}(t)\right|^{2}+\left|A^{1 / 2} u_{\varepsilon}(t)\right|^{2}\right)>0
$$


For the sake of simplicity we decided to state and prove our results in the model case $b(t)=(1+t)^{-1}$ with minimal assumptions on initial data. The same technique however can be applied with more regular initial data or more general dissipation terms. In this way one obtains the statements we mention in the two remarks below.

Remark 2.4 Let us assume that in Theorem 2.1 we have that $\left(u_{0}, u_{1}\right) \in D\left(A^{(k+1) / 2}\right) \times$ $D\left(A^{k / 2}\right)$ for some integer $k \geq 2$. Then it turns out that

$$
\begin{gathered}
(1+t)^{2 k}\left|A^{(k-1) / 2} u_{\varepsilon}^{\prime}(t)\right|^{2}+(1+t)^{2 k+2}\left(\varepsilon\left|A^{k / 2} u_{\varepsilon}^{\prime}(t)\right|^{2}+\left|A^{(k+1) / 2} u_{\varepsilon}(t)\right|^{2}\right) \leq C_{k}, \\
\int_{0}^{+\infty}(1+s)^{2 k+1}\left(\left|A^{k / 2} u_{\varepsilon}^{\prime}(s)\right|^{2}+\left|A^{(k+1) / 2} u_{\varepsilon}(s)\right|^{2}\right) d s \leq C_{k},
\end{gathered}
$$

where the constant $C_{k}$ depends on $k$, but not on $\varepsilon$ and $t$. The decay rates are the same obtained in (2.1) and (2.2) for the parabolic problem.

The decay-error estimates (2.9) through (2.13) admit similar extensions. In particular when $\left(u_{0}, u_{1}\right) \in D\left(A^{(k+2) / 2}\right) \times D\left(A^{k / 2}\right)$ for some integer $k \geq 2$ we have that

$$
\begin{gathered}
(1+t)^{k-1}\left|A^{(k-2) / 2} r_{\varepsilon}^{\prime}(t)\right|+(1+t)^{k}\left(\sqrt{\varepsilon}\left|A^{(k-1) / 2} r_{\varepsilon}^{\prime}(t)\right|+\left|A^{k / 2} \rho_{\varepsilon}(t)\right|\right) \leq C_{k} \varepsilon, \\
\int_{0}^{+\infty}(1+s)^{2 k-1}\left(\left|A^{k / 2} \rho_{\varepsilon}(s)\right|^{2}+\left|A^{(k-1) / 2} r_{\varepsilon}^{\prime}(s)\right|^{2}\right) d s \leq C_{k} \varepsilon^{2} .
\end{gathered}
$$

We sketch a proof of (2.16) and (2.17) in section 3.5.

Remark 2.5 Let us assume that $b(t)=1 / \varphi(t)$, where $\varphi:[0,+\infty) \rightarrow(0,+\infty)$ is any function such that $\varphi^{\prime}(t)$ is bounded, and

$$
\text { either } \int_{0}^{+\infty} \frac{\left[\varphi^{\prime}(s)\right]^{2}}{\varphi(s)} d s<+\infty \quad \text { or } \int_{0}^{+\infty}\left|\varphi^{\prime \prime}(s)\right| d s<+\infty \text {. }
$$

If $A, m, u_{0}, u_{1}$ are chosen as in Theorem 2.1, and

$$
\Phi(t):=1+\int_{0}^{t} \varphi(s) d s
$$

then we have that

$$
\begin{gathered}
\left|u_{\varepsilon}(t)\right|^{2}+\Phi(t)\left(\left|u_{\varepsilon}^{\prime}(t)\right|^{2}+\left|A^{1 / 2} u_{\varepsilon}(t)\right|^{2}\right)+\Phi^{2}(t)\left(\varepsilon\left|A^{1 / 2} u_{\varepsilon}^{\prime}(t)\right|^{2}+\left|A u_{\varepsilon}(t)\right|^{2}\right) \leq C, \\
\int_{0}^{+\infty} \varphi(s)\left[\left|u_{\varepsilon}^{\prime}(s)\right|^{2}+\left|A^{1 / 2} u_{\varepsilon}(s)\right|^{2}+\Phi(s)\left(\left|A^{1 / 2} u_{\varepsilon}^{\prime}(s)\right|^{2}+\left|A u_{\varepsilon}(s)\right|^{2}\right)\right] d s \leq C .
\end{gathered}
$$

These estimates generalize (2.3) through (2.8). Once again the decay rates of $u_{\varepsilon}(t)$ are exactly those expected for $u(t)$. The decay-error estimates (2.9) through (2.13) admit as well analogous extensions in terms of $\varphi(t)$ and $\Phi(t)$.

We don't prove these estimates explicitly because this generality only complicates proofs without introducing new ideas. 


\section{Proofs}

\subsection{Comparison results for ODEs}

In this section we state two comparison lemmata needed to prove our main results. We omit the simple standard proofs.

Lemma 3.1 Let $y:[0,+\infty) \rightarrow[0,+\infty)$ be a function of class $C^{1}$, and let $\psi:[0,+\infty) \rightarrow$ $[0,+\infty)$ be a continuous function. Let us assume that there exist two constants $c_{1}>0$ and $c_{2}>0$ such that

$$
y^{\prime}(t) \leq \psi(t)\left(-c_{1} y(t)+c_{2} \sqrt{y(t)}\right) \quad \forall t \geq 0 .
$$

Then $y(t) \leq \max \left\{y(0),\left(c_{2} / c_{1}\right)^{2}\right\}$ for every $t \geq 0$.

Lemma 3.2 For $i=1,2,3$ let $g_{i}:[0,+\infty) \rightarrow[0,+\infty)$ be a continuous function. Let $y:[0,+\infty) \rightarrow[0,+\infty)$ be a function of class $C^{1}$ such that $y(0)=0$ and

$$
y^{\prime}(t) \leq-g_{1}(t)+g_{2}(t) y(t)+g_{3}(t) \quad \forall t \geq 0 .
$$

For $i=1,2,3$ let us set

$$
G_{i}(t):=\int_{0}^{t} g_{i}(s) d s \quad \forall t \geq 0 .
$$

Then we have that

$$
y(t)+G_{1}(t) \leq e^{G_{2}(t)} G_{3}(t) \quad \forall t \geq 0 .
$$

\subsection{Decay estimates for the parabolic equation}

For every $k \in \mathbb{N}$ and every $t>0$ we have that

$$
\begin{aligned}
{\left[(1+t)^{2 k}\left|A^{k / 2} u(t)\right|^{2}\right]^{\prime}=} & 2 k(1+t)^{2 k-1}\left|A^{k / 2} u(t)\right|^{2}+ \\
& -2(1+t)^{2 k+1} m\left(\left|A^{1 / 2} u(t)\right|^{2}\right)\left|A^{(k+1) / 2} u(t)\right|^{2},
\end{aligned}
$$

hence

$$
\begin{gathered}
(1+t)^{2 k}\left|A^{k / 2} u(t)\right|^{2}+2 \int_{0}^{t}(1+s)^{2 k+1} m\left(\left|A^{1 / 2} u(s)\right|^{2}\right)\left|A^{(k+1) / 2} u(s)\right|^{2} d s \leq \\
\leq\left|A^{k / 2} u_{0}\right|^{2}+2 k \int_{0}^{t}(1+s)^{2 k-1}\left|A^{k / 2} u(s)\right|^{2} d s .
\end{gathered}
$$

Due to (1.1) this inequality implies (2.1) and (2.2) in the case $k=0$. For $k>0$ the same conclusions follow with an easy induction (we remind that all intermediate norms $\left|A^{\alpha} u_{0}\right|$, with $0 \leq \alpha \leq k / 2$, are controlled by $\left|u_{0}\right|$ and $\left.\left|A^{k / 2} u_{0}\right|\right)$. 


\subsection{Global existence and decay}

In this section we prove Theorem 2.1.

Local maximal solutions Problem (1.2), (1.3) admits a unique local-in-time solution, and this solution can be continued to a solution defined in a maximal interval $[0, T)$, where either $T=+\infty$, or

$$
\limsup _{t \rightarrow T^{-}}\left(\left|A^{1 / 2} u_{\varepsilon}^{\prime}(t)\right|^{2}+\left|A u_{\varepsilon}(t)\right|^{2}\right)=+\infty .
$$

We omit the proof of these standard results. The interested reader is referred to [5] (see also [1]).

Monotonicity of the Hamiltonian Let

$$
M(\sigma):=\int_{0}^{\sigma} m(s) d s,
$$

and let

$$
H(t):=\varepsilon\left|u_{\varepsilon}^{\prime}(t)\right|^{2}+M\left(\left|A^{1 / 2} u_{\varepsilon}(t)\right|^{2}\right)
$$

be the usual Hamiltonian. From (1.2) we have that

$$
H^{\prime}(t)=-2 \frac{\left|u_{\varepsilon}^{\prime}(t)\right|^{2}}{1+t} \quad \forall t \in[0, T),
$$

hence

$$
H(t)+2 \int_{0}^{t} \frac{\left|u_{\varepsilon}^{\prime}(s)\right|^{2}}{1+s} d s=H(0) \quad \forall t \in[0, T) .
$$

Since $M(\sigma) \geq \mu_{1} \sigma$ this implies that

$$
\left|A^{1 / 2} u_{\varepsilon}(t)\right|^{2} \leq \frac{H(0)}{\mu_{1}} \quad \forall t \in[0, T) .
$$

Definitions and preliminaries Let us set for simplicity

$$
c_{\varepsilon}(t):=m\left(\left|A^{1 / 2} u_{\varepsilon}(t)\right|^{2}\right) .
$$

Due to (1.1) and (3.5) we have that

$$
\mu_{1} \leq c_{\varepsilon}(t) \leq \mu_{2}
$$

where

$$
\mu_{2}:=\max \left\{m(\sigma): 0 \leq \sigma \leq \mu_{1}^{-1} H(0)\right\} .
$$


We also set

$$
L:=\max \left\{\left|m^{\prime}(\sigma)\right|: 0 \leq \sigma \leq \mu_{1}^{-1} H(0)\right\}
$$

so that

$$
\frac{\left|c_{\varepsilon}^{\prime}(t)\right|}{c_{\varepsilon}(t)} \leq \frac{2 L}{\mu_{1}}\left|\left\langle u^{\prime}(t), A u(t)\right\rangle\right| \quad \forall t \in[0, T) .
$$

Let us consider the following constants, depending only on $u_{0}, u_{1}$ (more precisely, on their norms in the spaces up to $\left.D(A) \times D\left(A^{1 / 2}\right)\right), \mu_{1}, \mu_{2}, L$ :

$$
\begin{gathered}
k_{1}:=\max \left\{2, \frac{1}{\mu_{1}}\right\}\left(\frac{\mu_{2}}{\mu_{1}}\left(\left|u_{1}\right|^{2}+\left|u_{0}\right|^{2}\right)+\left|u_{1}\right|^{2}+\mu_{2}\left|A^{1 / 2} u_{0}\right|^{2}\right), \\
k_{2}:=\max \left\{8, \frac{1}{\mu_{1}}\right\}\left(k_{1}+\left|u_{1}\right|^{2}+\left|u_{0}\right|^{2}\right), \\
k_{3}:=k_{2}+\frac{1}{2}\left(\left|A^{1 / 2} u_{0}\right|^{2}+\left|A^{1 / 2} u_{1}\right|^{2}\right), \\
k_{4}:=\max \left\{1,2 \mu_{2}\right\}\left(\frac{\left|A^{1 / 2} u_{1}\right|^{2}}{\mu_{1}}+\left|A u_{0}\right|^{2}+\frac{4}{\mu_{1}} k_{3}\right) .
\end{gathered}
$$

Finally, let $\varepsilon_{0}$ be small enough in such a way that

$$
\varepsilon_{0} \leq \min \left\{\frac{1}{8}, \frac{\mu_{1}}{8 \mu_{2}}\right\}, \quad \frac{2 L\left|\left\langle u_{1}, A u_{0}\right\rangle\right|}{\mu_{1}} \varepsilon_{0}<\frac{1}{2}, \quad \sqrt{\varepsilon_{0}} \leq \frac{\mu_{1}}{2 L\left(k_{1}+k_{4}\right)} .
$$

The core of this proof are some estimates on the following energies:

$$
\begin{aligned}
D_{\varepsilon, 0}(t) & :=\frac{1-\varepsilon}{2}\left|u_{\varepsilon}(t)\right|^{2}+\varepsilon(1+t)\left\langle u_{\varepsilon}^{\prime}(t), u_{\varepsilon}(t)\right\rangle \\
D_{\varepsilon, 1}(t) & :=\frac{1-3 \varepsilon}{2}(1+t)^{2}\left|A^{1 / 2} u_{\varepsilon}(t)\right|^{2}+\varepsilon(1+t)^{3}\left\langle u_{\varepsilon}^{\prime}(t), A u_{\varepsilon}(t)\right\rangle \\
F_{\varepsilon}(t) & :=\varepsilon \frac{\left|A^{1 / 2} u_{\varepsilon}^{\prime}(t)\right|^{2}}{c_{\varepsilon}(t)}+\left|A u_{\varepsilon}(t)\right|^{2} . \\
G_{\varepsilon}(t) & :=(1+t)^{2}\left|u_{\varepsilon}^{\prime}(t)\right|^{2}
\end{aligned}
$$

Integral estimate on $A^{1 / 2} u_{\varepsilon}$ The time derivative of (3.16) is

$$
D_{\varepsilon, 0}^{\prime}(t)=-(1+t) c_{\varepsilon}(t)\left|A^{1 / 2} u_{\varepsilon}(t)\right|^{2}+\varepsilon(1+t)\left|u_{\varepsilon}^{\prime}(t)\right|^{2} .
$$

Integrating in $[0, t]$ we obtain that

$$
\int_{0}^{t}(1+s) c_{\varepsilon}(s)\left|A^{1 / 2} u_{\varepsilon}(s)\right|^{2} d s=\varepsilon \int_{0}^{t}(1+s)\left|u_{\varepsilon}^{\prime}(s)\right|^{2} d s+D_{\varepsilon, 0}(0)-D_{\varepsilon, 0}(t) .
$$


Now we have that

$$
D_{\varepsilon, 0}(0)=\frac{1-\varepsilon}{2}\left|u_{0}\right|^{2}+\varepsilon\left\langle u_{0}, u_{1}\right\rangle \leq \frac{1}{2}\left|u_{0}\right|^{2}+\frac{\varepsilon}{2}\left|u_{1}\right|^{2},
$$

and, since $\varepsilon \leq 1 / 2$, we have that

$$
-D_{\varepsilon, 0}(t) \leq-\frac{1}{4}\left|u_{\varepsilon}(t)\right|^{2}+\varepsilon(1+t)\left|u_{\varepsilon}^{\prime}(t)\right| \cdot\left|u_{\varepsilon}(t)\right| \leq-\frac{1}{8}\left|u_{\varepsilon}(t)\right|^{2}+2 \varepsilon^{2}(1+t)^{2}\left|u_{\varepsilon}^{\prime}(t)\right|^{2} .
$$

Replacing these estimates in (3.20) we obtain that

$$
\begin{aligned}
\frac{1}{8}\left|u_{\varepsilon}(t)\right|^{2} & +\int_{0}^{t}(1+s) c_{\varepsilon}(s)\left|A^{1 / 2} u_{\varepsilon}(s)\right|^{2} d s \leq \\
\leq & \varepsilon \int_{0}^{t}(1+s)\left|u_{\varepsilon}^{\prime}(s)\right|^{2} d s+2 \varepsilon^{2}(1+t)^{2}\left|u_{\varepsilon}^{\prime}(t)\right|^{2}+\frac{1}{2}\left|u_{0}\right|^{2}+\frac{\varepsilon}{2}\left|u_{1}\right|^{2}
\end{aligned}
$$

First set of decay estimates We prove that

$$
\begin{gathered}
(1+t)^{2} \varepsilon\left|u_{\varepsilon}^{\prime}(t)\right|^{2}+(1+t)^{2}\left|A^{1 / 2} u_{\varepsilon}(t)\right|^{2}+\int_{0}^{t}(1+s)\left|u_{\varepsilon}^{\prime}(s)\right|^{2} d s \leq k_{1}, \\
\left|u_{\varepsilon}(t)\right|^{2}+\int_{0}^{t}(1+s)\left|A^{1 / 2} u_{\varepsilon}(s)\right|^{2} d s \leq k_{2},
\end{gathered}
$$

for every $t \in[0, T)$, where $k_{1}$ and $k_{2}$ are the constants defined by (3.11) and (3.12).

To this end from (3.4) we easily deduce that

$$
\left[(1+t)^{2} H(t)\right]^{\prime}=-2(1-\varepsilon)(1+t)\left|u_{\varepsilon}^{\prime}(t)\right|^{2}+2(1+t) M\left(\left|A^{1 / 2} u_{\varepsilon}(t)\right|^{2}\right) .
$$

Moreover from (3.5), (3.7), and (3.8) we have that

$$
M\left(\left|A^{1 / 2} u_{\varepsilon}(t)\right|^{2}\right) \leq \mu_{2}\left|A^{1 / 2} u_{\varepsilon}(t)\right|^{2} \leq \frac{\mu_{2}}{\mu_{1}} c_{\varepsilon}(t)\left|A^{1 / 2} u_{\varepsilon}(t)\right|^{2} .
$$

This allows to estimate the last term in (3.24), yielding that

$$
\left[(1+t)^{2} H(t)\right]^{\prime} \leq-2(1-\varepsilon)(1+t)\left|u_{\varepsilon}^{\prime}(t)\right|^{2}+\frac{2 \mu_{2}}{\mu_{1}}(1+t) c_{\varepsilon}(t)\left|A^{1 / 2} u_{\varepsilon}(t)\right|^{2} .
$$

Now we integrate in $[0, t]$ and we use (3.21). After rearranging the terms we obtain that

$$
\begin{gathered}
(1+t)^{2}\left(1-\frac{4 \mu_{2}}{\mu_{1}} \varepsilon\right) \varepsilon\left|u_{\varepsilon}^{\prime}(t)\right|^{2}+(1+t)^{2} M\left(\left|A^{1 / 2} u_{\varepsilon}(t)\right|^{2}\right)+ \\
+2\left(1-\varepsilon-\frac{\mu_{2}}{\mu_{1}} \varepsilon\right) \int_{0}^{t}(1+s)\left|u_{\varepsilon}^{\prime}(s)\right|^{2} d s \leq H(0)+\frac{\mu_{2}}{\mu_{1}}\left(\left|u_{0}\right|^{2}+\varepsilon\left|u_{1}\right|^{2}\right) .
\end{gathered}
$$


From our smallness assumptions on $\varepsilon$ and the usual estimates on $M\left(\left|A^{1 / 2} u_{\varepsilon}(t)\right|^{2}\right)$ we deduce that

$$
\begin{gathered}
\frac{1}{2}(1+t)^{2} \varepsilon\left|u_{\varepsilon}^{\prime}(t)\right|^{2}+\mu_{1}(1+t)^{2}\left|A^{1 / 2} u_{\varepsilon}(t)\right|^{2}+\int_{0}^{t}(1+s)\left|u_{\varepsilon}^{\prime}(s)\right|^{2} d s \leq \\
\leq\left|u_{1}\right|^{2}+\mu_{2}\left|A^{1 / 2} u_{0}\right|^{2}+\frac{\mu_{2}}{\mu_{1}}\left(\left|u_{0}\right|^{2}+\left|u_{1}\right|^{2}\right)
\end{gathered}
$$

from which (3.22) easily follows.

This allows to estimate the first two terms in the right-hand side of (3.21). We thus obtain (3.23).

Integral estimate on $A u_{\varepsilon}$ The time derivative of (3.17) is

$$
D_{\varepsilon, 1}^{\prime}(t)=-(1+t)^{3} c_{\varepsilon}(t)\left|A u_{\varepsilon}(t)\right|^{2}+\varepsilon(1+t)^{3}\left|A^{1 / 2} u_{\varepsilon}^{\prime}(t)\right|^{2}+(1-3 \varepsilon)(1+t)\left|A^{1 / 2} u_{\varepsilon}(t)\right|^{2} .
$$

Integrating in $[0, t]$ we obtain that

$$
\begin{aligned}
\int_{0}^{t}(1+s)^{3} c_{\varepsilon}(s)\left|A u_{\varepsilon}(s)\right|^{2} d s \leq & \varepsilon \int_{0}^{t}(1+s)^{3}\left|A^{1 / 2} u_{\varepsilon}^{\prime}(s)\right|^{2} d s+D_{\varepsilon, 1}(0)+ \\
& +\int_{0}^{t}(1+s)\left|A^{1 / 2} u_{\varepsilon}(s)\right|^{2} d s-D_{\varepsilon, 1}(t)
\end{aligned}
$$

The second integral in the right-hand side can be estimated using (3.23). Moreover

$$
D_{\varepsilon, 1}(0)=\frac{1-3 \varepsilon}{2}\left|A^{1 / 2} u_{0}\right|^{2}+\varepsilon\left\langle A^{1 / 2} u_{0}, A^{1 / 2} u_{1}\right\rangle \leq \frac{1}{2}\left|A^{1 / 2} u_{0}\right|^{2}+\frac{\varepsilon}{2}\left|A^{1 / 2} u_{1}\right|^{2},
$$

and, since $\varepsilon \leq 1 / 6$, we have that

$$
\begin{aligned}
-D_{\varepsilon, 1}(t) & \leq-\frac{1}{4}(1+t)^{2}\left|A^{1 / 2} u_{\varepsilon}(t)\right|^{2}+2 \cdot(1+t) \frac{\left|A^{1 / 2} u_{\varepsilon}(t)\right|}{2} \cdot \varepsilon(1+t)^{2}\left|A^{1 / 2} u_{\varepsilon}^{\prime}(t)\right| \\
& \leq \varepsilon^{2}(1+t)^{4}\left|A^{1 / 2} u_{\varepsilon}^{\prime}(t)\right|^{2} .
\end{aligned}
$$

Replacing all these estimates in (3.25) we obtain that

$$
\begin{aligned}
\int_{0}^{t}(1+s)^{3} c_{\varepsilon}(s)\left|A u_{\varepsilon}(s)\right|^{2} d s \leq \\
\quad \leq \varepsilon \int_{0}^{t}(1+s)^{3}\left|A^{1 / 2} u_{\varepsilon}^{\prime}(s)\right|^{2} d s+\varepsilon^{2}(1+t)^{4}\left|A^{1 / 2} u_{\varepsilon}^{\prime}(t)\right|^{2}+k_{3}
\end{aligned}
$$


Second set of decay estimates Let us set

$$
S:=\sup \left\{\tau \leq T: \varepsilon \frac{\left|c_{\varepsilon}^{\prime}(t)\right|}{c_{\varepsilon}(t)} \leq \frac{1}{2(1+t)} \quad \forall t \in[0, \tau]\right\} .
$$

We claim that $S>0$ and

$$
\begin{gathered}
(1+t)^{4} \varepsilon\left|A^{1 / 2} u_{\varepsilon}^{\prime}(t)\right|^{2}+(1+t)^{4}\left|A u_{\varepsilon}(t)\right|^{2}+\int_{0}^{t}(1+s)^{3}\left|A^{1 / 2} u_{\varepsilon}^{\prime}(s)\right|^{2} d s \leq k_{4}, \\
\int_{0}^{t}(1+s)^{3}\left|A u_{\varepsilon}(s)\right|^{2} d s \leq \frac{k_{4}+k_{3}}{\mu_{1}},
\end{gathered}
$$

for every $t \in[0, S)$, where $k_{3}$ and $k_{4}$ are the constants defined by (3.13) and (3.14).

Let us prove these claims. Thanks to inequality (3.10) with $t=0$ and the second inequality in (3.15) we have that

$$
\varepsilon \frac{\left|c_{\varepsilon}^{\prime}(0)\right|}{c_{\varepsilon}(0)} \leq \varepsilon_{0} \frac{2 L\left|\left\langle u_{1}, A u_{0}\right\rangle\right|}{\mu_{1}}<\frac{1}{2}
$$

hence $S>0$. Moreover we have that

$$
\left[(1+t)^{4} F_{\varepsilon}(t)\right]^{\prime}=-(1+t)^{4}\left(\frac{2-4 \varepsilon}{1+t}+\varepsilon \frac{c_{\varepsilon}^{\prime}(t)}{c_{\varepsilon}(t)}\right) \frac{\left|A^{1 / 2} u_{\varepsilon}^{\prime}(t)\right|^{2}}{c_{\varepsilon}(t)}+4(1+t)^{3}\left|A u_{\varepsilon}(t)\right|^{2}
$$

for every $t \in[0, T)$. Now let us restrict to $t \in[0, S)$. Due to the definition of $S$ and the fact that $\varepsilon \leq 1 / 8$ this implies that

$$
\left[(1+t)^{4} F_{\varepsilon}(t)\right]^{\prime} \leq-(1+t)^{3} \frac{\left|A^{1 / 2} u_{\varepsilon}^{\prime}(t)\right|^{2}}{c_{\varepsilon}(t)}+\frac{4}{\mu_{1}}(1+t)^{3} c_{\varepsilon}(t)\left|A u_{\varepsilon}(t)\right|^{2} .
$$

Now we integrate in $[0, t]$ and we use (3.26). After recollecting the terms we end up with

$$
\begin{aligned}
\left(\frac{1}{c_{\varepsilon}(t)}-\right. & \left.\frac{4}{\mu_{1}} \varepsilon\right)(1+t)^{4} \varepsilon\left|A^{1 / 2} u_{\varepsilon}^{\prime}(t)\right|^{2}+(1+t)^{4}\left|A u_{\varepsilon}(t)\right|^{2}+ \\
& +\int_{0}^{t}\left(\frac{1}{c_{\varepsilon}(s)}-\frac{4}{\mu_{1}} \varepsilon\right)(1+s)^{3}\left|A^{1 / 2} u_{\varepsilon}^{\prime}(s)\right|^{2} d s \leq F(0)+\frac{4}{\mu_{1}} k_{3} .
\end{aligned}
$$

Since $\varepsilon \leq \mu_{1} /\left(8 \mu_{2}\right)$ we have that

$$
\frac{1}{c_{\varepsilon}(t)}-\frac{4}{\mu_{1}} \varepsilon \geq \frac{1}{\mu_{2}}-\frac{4}{\mu_{1}} \varepsilon \geq \frac{1}{2 \mu_{2}}
$$

and therefore (3.28) easily follows from (3.30). This in turn allows to estimate the first two terms in the right-hand side of (3.26). We thus obtain (3.29). 
Global existence We prove that $S=T=+\infty$. Let us assume by contradiction that $S<T$. By definition (3.27) of $S$ this means that necessarily

$$
\varepsilon \frac{\left|c_{\varepsilon}^{\prime}(S)\right|}{c_{\varepsilon}(S)}=\frac{1}{2(1+S)}
$$

On the other hand, applying (3.10) with $t=S$, (3.22), (3.28), and the last inequality in (3.15), we have that

$$
\begin{aligned}
\varepsilon \frac{\left|c_{\varepsilon}^{\prime}(S)\right|}{c_{\varepsilon}(S)} \leq & \varepsilon \frac{2 L}{\mu_{1}}\left|\left\langle u_{\varepsilon}^{\prime}(S), A u_{\varepsilon}(S)\right\rangle\right| \leq \frac{L}{\mu_{1}} \sqrt{\varepsilon}\left(\varepsilon\left|u_{\varepsilon}^{\prime}(S)\right|^{2}+\left|A u_{\varepsilon}(S)\right|^{2}\right) \leq \\
& \leq \frac{L}{\mu_{1}} \sqrt{\varepsilon_{0}}\left(\frac{k_{1}}{(1+S)^{2}}+\frac{k_{4}}{(1+S)^{4}}\right)<\frac{1}{2(1+S)}
\end{aligned}
$$

which contradicts $(3.31)$.

It remains to prove that $T=+\infty$. To this end it is enough to show that (3.2) cannot be true, and this immediately follows from (3.28).

Last decay estimate Since $T=+\infty$ we know that (3.22) and (3.23) hold true for every $t \geq 0$. This proves (2.3), (2.4), and (2.6). Since $S=+\infty$ we know that also (3.28) and (3.29) hold true for every $t \geq 0$. This proves (2.7) and (2.8).

It remains to prove (2.5). To this end we compute the time derivative of (3.19):

$$
G_{\varepsilon}^{\prime}(t)=-2\left(\frac{1}{\varepsilon}-1\right)(1+t)\left|u_{\varepsilon}^{\prime}(t)\right|^{2}-\frac{2}{\varepsilon}(1+t)^{2}\left\langle u_{\varepsilon}^{\prime}(t), c_{\varepsilon}(t) A u_{\varepsilon}(t)\right\rangle .
$$

From estimate (3.28) and the fact that $\varepsilon \leq 1 / 2$ it follows that

$$
\begin{aligned}
G_{\varepsilon}^{\prime}(t) & \leq-\frac{G_{\varepsilon}(t)}{\varepsilon(1+t)}+\frac{2 \mu_{2}}{\varepsilon}(1+t)\left|A u_{\varepsilon}(t)\right| \sqrt{G_{\varepsilon}(t)} \\
& \leq \frac{1}{\varepsilon(1+t)}\left(-G_{\varepsilon}(t)+2 \mu_{2} \sqrt{k_{4}} \sqrt{G_{\varepsilon}(t)}\right) .
\end{aligned}
$$

Therefore the conclusion follows from Lemma 3.1 applied with $y(t):=G_{\varepsilon}(t)$.

\subsection{Decay-error estimates}

In this section we prove Theorem 2.2. 
Notations and preliminaries Throughout this proof we assume that $\varepsilon \in\left(0, \varepsilon_{0}\right)$, where $\varepsilon_{0}$ satisfies inequalities (3.15) as in the proof of the global existence result, and the further inequality

$$
\varepsilon_{0} \leq \frac{\mu_{1}}{128 \mu_{2}}
$$

Under assumption (3.15) we already know that problem (1.2), (1.3) has a unique global solution satisfying (2.3) through (2.8), and

$$
\varepsilon \frac{\left|c_{\varepsilon}^{\prime}(t)\right|}{c_{\varepsilon}(t)} \leq \frac{1}{2(1+t)} \quad \forall t \geq 0
$$

where of course $c_{\varepsilon}(t)$ is defined by (3.6). Accordingly we set $c(t):=m\left(\left|A^{1 / 2} u(t)\right|^{2}\right)$. The function $t \rightarrow M\left(\left|A^{1 / 2} u(t)\right|^{2}\right)$ turns out to be nonincreasing, and therefore inequalities (3.5) and (3.7) hold true also with $u(t)$ and $c(t)$ in place of $u_{\varepsilon}(t)$ and $c_{\varepsilon}(t)$.

The corrector $\theta_{\varepsilon}(t)$, namely the solution of (1.6), (1.7), can be explicitly computed to be

$$
\theta_{\varepsilon}(t)=\frac{\varepsilon}{1-\varepsilon}\left(1-(1+t)^{1-1 / \varepsilon}\right) w_{0}
$$

so that

$$
\theta_{\varepsilon}^{\prime}(t)=w_{0}(1+t)^{-1 / \varepsilon}
$$

Simple calculations show that $r_{\varepsilon}$ is the solution of the Cauchy problem

$$
\begin{gathered}
\varepsilon r_{\varepsilon}^{\prime \prime}(t)+\frac{1}{1+t} r_{\varepsilon}^{\prime}(t)+c_{\varepsilon}(t) A \rho_{\varepsilon}(t)=\left(c(t)-c_{\varepsilon}(t)\right) A u(t)-\varepsilon u^{\prime \prime}(t), \\
r_{\varepsilon}(0)=0, \quad r_{\varepsilon}^{\prime}(0)=0,
\end{gathered}
$$

while $\rho_{\varepsilon}$ is the solution of the Cauchy problem

$$
\begin{gathered}
\varepsilon \rho_{\varepsilon}^{\prime \prime}(t)+\frac{1}{1+t} \rho_{\varepsilon}^{\prime}(t)+c_{\varepsilon}(t) A \rho_{\varepsilon}(t)=\left(c(t)-c_{\varepsilon}(t)\right) A u(t)-\varepsilon u^{\prime \prime}(t), \\
\rho_{\varepsilon}(0)=0, \quad \rho_{\varepsilon}^{\prime}(0)=w_{0} .
\end{gathered}
$$

In order to estimate $\rho_{\varepsilon}$ and $r_{\varepsilon}$ we introduce the energies

$$
\begin{gathered}
\mathcal{D}_{\varepsilon}(t):=\frac{1-\varepsilon}{2}\left|\rho_{\varepsilon}(t)\right|^{2}+\varepsilon(1+t)\left\langle\rho_{\varepsilon}^{\prime}(t), \rho_{\varepsilon}(t)\right\rangle, \\
\mathcal{E}_{\varepsilon}(t):=\varepsilon \frac{\left|r_{\varepsilon}^{\prime}(t)\right|^{2}}{c_{\varepsilon}(t)}+\left|A^{1 / 2} \rho_{\varepsilon}\right|^{2} .
\end{gathered}
$$

From now on $K_{1}, K_{2}, \alpha_{1}, \ldots, \alpha_{23}$ denote constants depending only on $u_{0}, u_{1}$ (more precisely on their norms in the spaces up to $\left.D\left(A^{3 / 2}\right) \times D\left(A^{1 / 2}\right)\right), \mu_{1}, \mu_{2}, L$. 
Integral estimate on $u^{\prime \prime}$ We prove that when $u_{0} \in D\left(A^{3 / 2}\right)$ we have that

$$
\int_{0}^{+\infty}(1+s)^{3}\left|u^{\prime \prime}(s)\right|^{2} d s<+\infty
$$

Indeed for every $t>0$ we have that

$$
u^{\prime \prime}(t)=-c(t) A u(t)+(1+t)^{2} c^{2}(t) A^{2} u(t)+2(1+t)^{2} c(t) m^{\prime}\left(\left|A^{1 / 2} u(t)\right|^{2}\right)|A u(t)|^{2} A u(t) .
$$

From (2.1) with $k=1$ we deduce that $\left|A^{1 / 2} u(t)\right|$ is bounded, hence also $c(t)$ and $m^{\prime}\left(\left|A^{1 / 2} u(t)\right|^{2}\right)$ are bounded. Moreover also $(1+t)^{2}|A u(t)|^{2}$ is bounded because of $(2.1)$ with $k=2$. It follows that

$$
\left|u^{\prime \prime}(t)\right| \leq \alpha_{1}|A u(t)|+\alpha_{2}(1+t)^{2}\left|A^{2} u(t)\right|
$$

hence

$$
(1+t)^{3}\left|u^{\prime \prime}(t)\right|^{2} \leq \alpha_{3}(1+t)^{3}|A u(t)|^{2}+\alpha_{4}(1+t)^{7}\left|A^{2} u(t)\right|^{2} .
$$

Therefore (3.38) follows from (2.2) applied with $k=1$ and $k=3$.

Boundedness of $\mathcal{E}_{\varepsilon} \quad$ We prove that there exists a constant $K_{1}$ such that

$$
\mathcal{E}_{\varepsilon}(t)+\int_{0}^{t} \frac{1}{1+s} \frac{\left|r_{\varepsilon}^{\prime}(s)\right|^{2}}{c_{\varepsilon}(s)} d s \leq K_{1} \varepsilon^{2}, \quad \forall t \geq 0
$$

The time derivative of $(3.37)$ is

$$
\begin{aligned}
\mathcal{E}_{\varepsilon}^{\prime}(t)= & -\frac{\left|r_{\varepsilon}^{\prime}(t)\right|^{2}}{c_{\varepsilon}(t)}\left(\varepsilon \frac{c_{\varepsilon}^{\prime}(t)}{c_{\varepsilon}(t)}+\frac{2}{1+t}\right)+2\left\langle\theta_{\varepsilon}^{\prime}(t), A \rho_{\varepsilon}(t)\right\rangle+ \\
& +\frac{2}{c_{\varepsilon}(t)}\left\langle r_{\varepsilon}^{\prime}(t),\left(c(t)-c_{\varepsilon}(t)\right) A u(t)-\varepsilon u^{\prime \prime}(t)\right\rangle .
\end{aligned}
$$

Let us estimate the three terms in the right-hand side. The first summand can be easily estimated by (3.33). For the second summand we use (3.35) and we obtain that

$$
2\left\langle\theta_{\varepsilon}^{\prime}(t), A \rho_{\varepsilon}(t)\right\rangle \leq 2\left|A^{1 / 2} \theta_{\varepsilon}^{\prime}(t)\right| \cdot\left|A^{1 / 2} \rho_{\varepsilon}(t)\right| \leq 2\left|A^{1 / 2} w_{0}\right|(1+t)^{-1 / \varepsilon}\left|A^{1 / 2} \rho_{\varepsilon}(t)\right| .
$$

From (3.9), (2.1) with $k=1$, and (2.4) we have that

$$
\begin{aligned}
\left|c(t)-c_{\varepsilon}(t)\right| & \leq\left. L|| A^{1 / 2} u(t)\right|^{2}-\left|A^{1 / 2} u_{\varepsilon}(t)\right|^{2} \mid \\
& \leq L\left(\left|A^{1 / 2} u(t)\right|+\left|A^{1 / 2} u_{\varepsilon}(t)\right|\right)\left|A^{1 / 2} \rho_{\varepsilon}(t)\right| \\
& \leq \alpha_{5}\left|A^{1 / 2} \rho_{\varepsilon}(t)\right|,
\end{aligned}
$$


hence (for shortness' sake we omit the dependence on $t$ )

$$
\begin{aligned}
\frac{2}{c_{\varepsilon}}\left\langle r_{\varepsilon}^{\prime},\left(c-c_{\varepsilon}\right) A u-\varepsilon u^{\prime \prime}\right\rangle & \leq \frac{1}{c_{\varepsilon}} \cdot 2 \frac{\left|r_{\varepsilon}^{\prime}\right|}{\sqrt{1+t}} \cdot \sqrt{1+t}\left(\left|c-c_{\varepsilon}\right||A u|+\varepsilon\left|u^{\prime \prime}\right|\right) \\
& \leq \frac{1}{2} \frac{\left|r_{\varepsilon}^{\prime}\right|^{2}}{(1+t) c_{\varepsilon}}+\frac{4}{c_{\varepsilon}}(1+t)\left(\left|c-c_{\varepsilon}\right|^{2}|A u|^{2}+\varepsilon^{2}\left|u^{\prime \prime}\right|^{2}\right) \\
& \leq \frac{1}{2} \frac{\left|r_{\varepsilon}^{\prime}\right|^{2}}{(1+t) c_{\varepsilon}}+\alpha_{6}(1+t)\left|A^{1 / 2} \rho_{\varepsilon}\right|^{2}|A u|^{2}+\alpha_{7} \varepsilon^{2}(1+t)\left|u^{\prime \prime}\right|^{2} .
\end{aligned}
$$

Replacing all these estimates in (3.40) we obtain that

$$
\begin{aligned}
\mathcal{E}_{\varepsilon}^{\prime}(t) \leq & -\frac{1}{1+t} \frac{\left|r_{\varepsilon}^{\prime}(t)\right|^{2}}{c_{\varepsilon}(t)}+\alpha_{6}(1+t)|A u(t)|^{2}\left|A^{1 / 2} \rho_{\varepsilon}(t)\right|^{2}+ \\
& +\alpha_{7}(1+t) \varepsilon^{2}\left|u^{\prime \prime}(t)\right|^{2}+\alpha_{8}(1+t)^{-1 / \varepsilon}\left|A^{1 / 2} \rho_{\varepsilon}(t)\right|,
\end{aligned}
$$

and in particular

$$
\begin{aligned}
\mathcal{E}_{\varepsilon}^{\prime}(t) \leq & -\frac{1}{1+t} \frac{\left|r_{\varepsilon}^{\prime}(t)\right|^{2}}{c_{\varepsilon}(t)}+\alpha_{6}(1+t)|A u(t)|^{2} \mathcal{E}_{\varepsilon}(t)+ \\
& +\alpha_{7}(1+t) \varepsilon^{2}\left|u^{\prime \prime}(t)\right|^{2}+\alpha_{8}(1+t)^{-1 / \varepsilon} \sup _{t \geq 0}\left|A^{1 / 2} \rho_{\varepsilon}(t)\right| . \\
=: & -g_{1}(t)+g_{2}(t) \mathcal{E}_{\varepsilon}(t)+g_{3}(t) .
\end{aligned}
$$

We can now apply Lemma 3.2 with $y(t):=\mathcal{E}_{\varepsilon}(t)$ (we recall that $\mathcal{E}_{\varepsilon}(0)=0$ ). The function $G_{2}(t)$ defined according to (3.1) is bounded in $t$ because of (2.2) with $k=1$. It follows that

$$
\begin{aligned}
\mathcal{E}_{\varepsilon}(t)+\int_{0}^{t} \frac{1}{1+s} \frac{\left|r_{\varepsilon}^{\prime}(s)\right|^{2}}{c_{\varepsilon}(s)} d s \leq & \alpha_{9} \varepsilon^{2} \int_{0}^{t}(1+s)\left|u^{\prime \prime}(s)\right|^{2} d s+ \\
& +\alpha_{9} \sup _{t \geq 0}\left|A^{1 / 2} \rho_{\varepsilon}(t)\right| \int_{0}^{t}(1+s)^{-1 / \varepsilon} d s .
\end{aligned}
$$

The first integral in the right-hand side is uniformly bounded due to (3.38), the second one is less than $2 \varepsilon$. It follows that

$$
\mathcal{E}_{\varepsilon}(t)+\int_{0}^{t} \frac{1}{1+s} \frac{\left|r_{\varepsilon}^{\prime}(s)\right|^{2}}{c_{\varepsilon}(s)} d s \leq \alpha_{10} \varepsilon^{2}+\alpha_{11} \varepsilon \sup _{t \geq 0}\left|A^{1 / 2} \rho_{\varepsilon}(t)\right|
$$

In particular

$$
\left(\sup _{t \geq 0}\left|A^{1 / 2} \rho_{\varepsilon}(t)\right|\right)^{2} \leq \alpha_{10} \varepsilon^{2}+\alpha_{11} \varepsilon \sup _{t \geq 0}\left|A^{1 / 2} \rho_{\varepsilon}(t)\right| \leq \frac{1}{2}\left(\sup _{t \geq 0}\left|A^{1 / 2} \rho_{\varepsilon}(t)\right|\right)^{2}+\alpha_{12} \varepsilon^{2},
$$

and therefore

$$
\sup _{t \geq 0}\left|A^{1 / 2} \rho_{\varepsilon}(t)\right| \leq \alpha_{13} \varepsilon
$$

Coming back to (3.43) we obtain (3.39). 
Integral estimate on $A^{1 / 2} \rho_{\varepsilon}$ The time derivative of (3.36) is

$$
\begin{aligned}
\mathcal{D}_{\varepsilon}^{\prime}(t)= & -(1+t) c_{\varepsilon}(t)\left|A^{1 / 2} \rho_{\varepsilon}(t)\right|^{2}+\varepsilon(1+t)\left|\rho_{\varepsilon}^{\prime}(t)\right|^{2}+ \\
& +(1+t)\left(c(t)-c_{\varepsilon}(t)\right)\left\langle A u(t), \rho_{\varepsilon}(t)\right\rangle-\varepsilon(1+t)\left\langle u^{\prime \prime}(t), \rho_{\varepsilon}(t)\right\rangle .
\end{aligned}
$$

Let us estimate the last three terms. From (3.35) we have that

$$
\left|\rho_{\varepsilon}^{\prime}(t)\right|^{2} \leq 2\left(\left|r_{\varepsilon}^{\prime}(t)\right|^{2}+\left|\theta_{\varepsilon}^{\prime}(t)\right|^{2}\right) \leq 2\left|r_{\varepsilon}^{\prime}(t)\right|^{2}+\alpha_{14}(1+t)^{-2 / \varepsilon} .
$$

Moreover from (3.41) and (3.44) we deduce that

$$
\begin{aligned}
\left(c(t)-c_{\varepsilon}(t)\right)\left\langle A u(t), \rho_{\varepsilon}(t)\right\rangle & \leq\left|c(t)-c_{\varepsilon}(t)\right| \cdot\left|A^{1 / 2} u(t)\right| \cdot\left|A^{1 / 2} \rho_{\varepsilon}(t)\right| \\
& \leq\left|A^{1 / 2} \rho_{\varepsilon}(t)\right| \cdot\left(\alpha_{5}\left|A^{1 / 2} \rho_{\varepsilon}(t)\right| \cdot\left|A^{1 / 2} u(t)\right|\right) \\
& \leq \frac{1}{4} c_{\varepsilon}(t)\left|A^{1 / 2} \rho_{\varepsilon}(t)\right|^{2}+\frac{\alpha_{5}^{2}}{c_{\varepsilon}(t)}\left|A^{1 / 2} \rho_{\varepsilon}(t)\right|^{2}\left|A^{1 / 2} u(t)\right|^{2} \\
& \leq \frac{1}{4} c_{\varepsilon}(t)\left|A^{1 / 2} \rho_{\varepsilon}(t)\right|^{2}+\alpha_{15} \varepsilon^{2}\left|A^{1 / 2} u(t)\right|^{2} .
\end{aligned}
$$

The term involving $u^{\prime \prime}(t)$ can be estimated more or less as in the proof of (3.38). We obtain that

$$
\begin{aligned}
\left|\varepsilon\left\langle\rho_{\varepsilon}(t), u^{\prime \prime}(t)\right\rangle\right| & \leq\left|A^{1 / 2} \rho_{\varepsilon}(t)\right| \cdot \varepsilon\left(\alpha_{1}\left|A^{1 / 2} u(t)\right|+\alpha_{2}(1+t)^{2}\left|A^{3 / 2} u(t)\right|\right) \\
& \leq \frac{1}{4} c_{\varepsilon}(t)\left|A^{1 / 2} \rho_{\varepsilon}(t)\right|^{2}+\alpha_{16} \varepsilon^{2}\left(\left|A^{1 / 2} u(t)\right|^{2}+(1+t)^{4}\left|A^{3 / 2} u(t)\right|^{2}\right) .
\end{aligned}
$$

Replacing all these estimates in the expression for $\mathcal{D}_{\varepsilon}^{\prime}(t)$ we find that

$$
\begin{aligned}
\mathcal{D}_{\varepsilon}^{\prime}(t) \leq & -\frac{1}{2}(1+t) c_{\varepsilon}(t)\left|A^{1 / 2} \rho_{\varepsilon}(t)\right|^{2}+2 \varepsilon(1+t)\left|r_{\varepsilon}^{\prime}(t)\right|^{2}+\alpha_{14} \varepsilon(1+t)^{1-2 / \varepsilon}+ \\
& +\alpha_{17} \varepsilon^{2}(1+t)\left|A^{1 / 2} u(t)\right|^{2}+\alpha_{16} \varepsilon^{2}(1+t)^{5}\left|A^{3 / 2} u(t)\right|^{2} .
\end{aligned}
$$

Now we integrate in $[0, t]$. Since $\mathcal{D}_{\varepsilon}(0)=0$, by $(2.2)$ with $k=0$ and $k=2$ we obtain that

$$
\frac{1}{2} \int_{0}^{t}(1+s) c_{\varepsilon}(s)\left|A^{1 / 2} \rho_{\varepsilon}(s)\right|^{2} d s \leq 2 \varepsilon \int_{0}^{t}(1+s)\left|r_{\varepsilon}^{\prime}(s)\right|^{2} d s+\alpha_{18} \varepsilon^{2}-\mathcal{D}_{\varepsilon}(t) .
$$

Let us estimate the last term. By the smallness of $\varepsilon$ and (3.35) we have that

$$
\begin{aligned}
-\mathcal{D}_{\varepsilon}(t) & \leq-\frac{1}{4}\left|\rho_{\varepsilon}(t)\right|^{2}+\left|\rho_{\varepsilon}(t)\right| \cdot \varepsilon(1+t)\left|\rho_{\varepsilon}^{\prime}(t)\right| \\
& \leq-\frac{1}{8}\left|\rho_{\varepsilon}(t)\right|^{2}+4 \varepsilon^{2}(1+t)^{2}\left(\left|r_{\varepsilon}^{\prime}(t)\right|^{2}+\left|\theta_{\varepsilon}^{\prime}(t)\right|^{2}\right) \\
& \leq-\frac{1}{8}\left|\rho_{\varepsilon}(t)\right|^{2}+4 \varepsilon^{2}(1+t)^{2}\left|r_{\varepsilon}^{\prime}(t)\right|^{2}+\alpha_{19} \varepsilon^{2} .
\end{aligned}
$$


Going back to (3.45) this implies that

$$
\begin{aligned}
\left|\rho_{\varepsilon}(t)\right|^{2} & +\int_{0}^{t}(1+s) c_{\varepsilon}(s)\left|A^{1 / 2} \rho_{\varepsilon}(s)\right|^{2} d s \leq \\
& \leq 16 \varepsilon \int_{0}^{t}(1+s)\left|r_{\varepsilon}^{\prime}(s)\right|^{2} d s+32 \varepsilon^{2}(1+t)^{2}\left|r_{\varepsilon}^{\prime}(t)\right|^{2} d s+\alpha_{20} \varepsilon^{2} .
\end{aligned}
$$

Decay estimates on $\mathcal{E}_{\varepsilon}$ We improve (3.39) and (3.46) by showing that there exists a constant $K_{2}$ such that

$$
\begin{gathered}
(1+t)^{2} \mathcal{E}_{\varepsilon}(t)+\int_{0}^{+\infty}(1+s)\left|r_{\varepsilon}^{\prime}(s)\right|^{2} d s \leq K_{2} \varepsilon^{2}, \quad \forall t \geq 0, \\
\left|\rho_{\varepsilon}(t)\right|^{2}+\int_{0}^{t}(1+s)\left|A^{1 / 2} \rho_{\varepsilon}(s)\right|^{2} d s \leq K_{2} \varepsilon^{2} \quad \forall t \geq 0 .
\end{gathered}
$$

These estimates imply (2.9) through (2.13).

From (3.42) we have that

$$
\begin{aligned}
{\left[(1+t)^{2} \mathcal{E}_{\varepsilon}(t)\right]^{\prime}=} & (1+t)^{2} \mathcal{E}_{\varepsilon}^{\prime}(t)+2(1+t) \mathcal{E}_{\varepsilon}(t) \\
\leq & -(1-2 \varepsilon)(1+t) \frac{\left|r_{\varepsilon}^{\prime}(t)\right|^{2}}{c_{\varepsilon}(t)}+2(1+t)\left|A^{1 / 2} \rho_{\varepsilon}(t)\right|^{2}+ \\
& +(1+t)^{3}\left(\alpha_{6}|A u(t)|^{2}\left|A^{1 / 2} \rho_{\varepsilon}(t)\right|^{2}+\alpha_{7} \varepsilon^{2}\left|u^{\prime \prime}(t)\right|^{2}\right)+ \\
& +\alpha_{8}(1+t)^{2-1 / \varepsilon}\left|A^{1 / 2} \rho_{\varepsilon}(t)\right| .
\end{aligned}
$$

By the smallness assumptions on $\varepsilon$ and estimate (3.44) this implies that

$$
\begin{aligned}
{\left[(1+t)^{2} \mathcal{E}_{\varepsilon}(t)\right]^{\prime} \leq } & -\frac{1}{2}(1+t) \frac{\left|r_{\varepsilon}^{\prime}(t)\right|^{2}}{c_{\varepsilon}(t)}+\frac{2}{\mu_{1}}(1+t) c_{\varepsilon}(t)\left|A^{1 / 2} \rho_{\varepsilon}(t)\right|^{2}+ \\
& +\alpha_{21} \varepsilon^{2}(1+t)^{3}\left(|A u(t)|^{2}+\left|u^{\prime \prime}(t)\right|^{2}\right)+\alpha_{22} \varepsilon(1+t)^{2-1 / \varepsilon} .
\end{aligned}
$$

Now we integrate in $[0, t]$. By $(3.46),(2.2)$ with $k=1$, and (3.38) we obtain that

$$
\begin{aligned}
& (1+t)^{2} \mathcal{E}_{\varepsilon}(t)+\frac{1}{2} \int_{0}^{t}(1+s) \frac{\left|r_{\varepsilon}^{\prime}(s)\right|^{2}}{c_{\varepsilon}(s)} d s \leq \\
& \leq \alpha_{23} \varepsilon^{2}+\frac{32}{\mu_{1}} \varepsilon \int_{0}^{t}(1+s)\left|r_{\varepsilon}^{\prime}(s)\right|^{2} d s+\frac{64}{\mu_{1}} \varepsilon^{2}(1+t)^{2}\left|r_{\varepsilon}^{\prime}(t)\right|^{2} .
\end{aligned}
$$

Rearranging the terms this may be rewritten as

$$
\begin{aligned}
\left(\frac{1}{c_{\varepsilon}(t)}-\frac{64}{\mu_{1}} \varepsilon\right) & (1+t)^{2} \varepsilon\left|r_{\varepsilon}^{\prime}(t)\right|^{2}+(1+t)^{2}\left|A^{1 / 2} \rho_{\varepsilon}(t)\right|^{2}+ \\
& +\frac{1}{2} \int_{0}^{t}\left(\frac{1}{c_{\varepsilon}(t)}-\frac{64}{\mu_{1}} \varepsilon\right)(1+s)\left|r_{\varepsilon}^{\prime}(s)\right|^{2} d s \leq \alpha_{22} \varepsilon^{2}
\end{aligned}
$$


By (3.32) we have that

$$
\frac{1}{c_{\varepsilon}(t)}-\frac{64}{\mu_{1}} \varepsilon \geq \frac{1}{\mu_{2}}-\frac{64}{\mu_{1}} \varepsilon \geq \frac{1}{2 \mu_{2}}
$$

and therefore (3.47) easily follows from (3.49).

This in turn allows to estimate the first two terms in the right-hand side of (3.46). We thus obtain (3.48).

\subsection{Decay-error estimates for more regular data}

Let us assume that $\left(u_{0}, u_{1}\right) \in D\left(A^{(k+2) / 2}\right) \times D\left(A^{k / 2}\right)$. Let us consider the energies

$$
\begin{gathered}
\mathcal{D}_{\varepsilon, k}(t):=\frac{1-(2 k-1) \varepsilon}{2}(1+t)^{2 k-2}\left|A^{(k-1) / 2} \rho_{\varepsilon}(t)\right|^{2}+\varepsilon(1+t)^{2 k-1}\left\langle A^{(k-1) / 2} \rho_{\varepsilon}^{\prime}(t), A^{(k-1) / 2} \rho_{\varepsilon}(t)\right\rangle, \\
\mathcal{E}_{\varepsilon, k}(t):=\varepsilon \frac{\left|A^{(k-1) / 2} r_{\varepsilon}^{\prime}(t)\right|^{2}}{c_{\varepsilon}(t)}+\left|A^{k / 2} \rho_{\varepsilon}(t)\right|^{2},
\end{gathered}
$$

which are the natural extensions of those defined in (3.36) and (3.37). For simplicity in this section $C_{k}$ denotes a constant, which may be different from line to line, but always depends only on $k$, on $\mu_{1}, \mu_{2}, L$, and on the norms of initial data in the appropriate spaces (so that $C_{k}$ doesn't depend on $\varepsilon$ and $t$ ).

Working with $\mathcal{D}_{\varepsilon, k}(t)$ and $(1+t)^{2 k} \mathcal{E}_{\varepsilon, k}(t)$ as we did with $\mathcal{D}_{\varepsilon}(t)$ and $(1+t)^{2} \mathcal{E}_{\varepsilon}(t)$, with an easy induction we obtain (2.17) and

$$
(1+t)^{k}\left(\sqrt{\varepsilon}\left|A^{(k-1) / 2} r_{\varepsilon}^{\prime}(t)\right|+\left|A^{k / 2} \rho_{\varepsilon}(t)\right|\right) \leq C_{k} \varepsilon .
$$

In order to prove (2.16) it remains to show that

$$
(1+t)^{k-1}\left|A^{(k-2) / 2} r_{\varepsilon}^{\prime}(t)\right| \leq C_{k} \varepsilon .
$$

To this end we consider the energy

$$
\mathcal{G}_{\varepsilon, k}(t):=(1+t)^{2 k-2}\left|A^{(k-2) / 2} r_{\varepsilon}^{\prime}(t)\right|^{2},
$$

which is the natural extension of (3.19). Its time derivative is (for shortness' sake we omit the dependence on $t$ )

$$
\begin{aligned}
\mathcal{G}_{\varepsilon, k}^{\prime}= & \left(2 k-2-\frac{2}{\varepsilon}\right)(1+t)^{2 k-3}\left|A^{(k-2) / 2} r_{\varepsilon}^{\prime}\right|^{2}+ \\
& -\frac{2}{\varepsilon}(1+t)^{2 k-2}\left\langle A^{(k-2) / 2} r_{\varepsilon}^{\prime}, c_{\varepsilon} A^{k / 2} \rho_{\varepsilon}+\left(c_{\varepsilon}-c\right) A^{k / 2} u+\varepsilon A^{(k-2) / 2} u^{\prime \prime}\right\rangle \\
\leq & \left(2 k-2-\frac{2}{\varepsilon}\right) \frac{1}{1+t} \mathcal{G}_{\varepsilon, k}+\frac{2}{\varepsilon} \frac{1}{1+t} \sqrt{\mathcal{G}_{\varepsilon, k}} \times \\
& \times(1+t)^{k}\left\{c_{\varepsilon}\left|A^{k / 2} \rho_{\varepsilon}\right|+\left|c_{\varepsilon}-c\right|\left|A^{k / 2} u\right|+\varepsilon\left|A^{(k-2) / 2} u^{\prime \prime}\right|\right\} .
\end{aligned}
$$


All the terms in the last line can be easily estimated. From (3.50), (2.1), (3.41), and (3.44) we have indeed that

$$
(1+t)^{k}\left|A^{k / 2} \rho_{\varepsilon}(t)\right| \leq C_{k} \varepsilon, \quad\left|c_{\varepsilon}(t)-c(t)\right|(1+t)^{k}\left|A^{k / 2} u(t)\right| \leq C_{k} \varepsilon,
$$

while arguing as in the proof of (3.38) we obtain that

$$
(1+t)^{k}\left|A^{(k-2) / 2} u^{\prime \prime}(t)\right| \leq \alpha_{1}(1+t)^{k}\left|A^{k / 2} u(t)\right|+\alpha_{2}(1+t)^{k+2}\left|A^{(k+2) / 2} u(t)\right| \leq C_{k} .
$$

Therefore for $\varepsilon$ small enough it turns out that

$$
\mathcal{G}_{\varepsilon, k}^{\prime}(t) \leq \frac{1}{\varepsilon(1+t)}\left(-\mathcal{G}_{\varepsilon, k}(t)+\varepsilon C_{k} \sqrt{\mathcal{G}_{\varepsilon, k}(t)}\right) \quad \forall t \geq 0
$$

Since $\mathcal{G}_{\varepsilon, k}(0)=0$, from Lemma 3.1 we deduce that $\mathcal{G}_{\varepsilon, k}(t) \leq C_{k}^{2} \varepsilon^{2}$ for every $t \geq 0$, which is equivalent to $(3.51)$.

\subsection{The supercritical case}

Let us prove Theorem 2.3. Let us define $H(t)$ as in (3.3). Then

$$
H^{\prime}(t)=-2 b(t)\left|u_{\varepsilon}^{\prime}(t)\right|^{2} \geq-\frac{2}{\varepsilon} b(t) H(t)
$$

hence

$$
H(t) \geq H(0) \exp \left(-\frac{2}{\varepsilon} \int_{0}^{t} b(s) d s\right) .
$$

The right-hand side is greater than a positive constant independent on $t$ because of (2.14) and the fact that $H(0)>0$. This implies (2.15).

\section{Acknowledgements}

We would like to thank Professor Taeko Yamazaki for sending us a preliminary version of references [18] and [19], and for pointing out reference [15].

\section{References}

[1] A. Arosio, S. Garavaldi; On the mildly degenerate Kirchhoff string. Math. Methods Appl. Sci. 14 (1991), no. 3, 177-195.

[2] E. H. DE BRITO; The damped elastic stretched string equation generalized: existence, uniqueness, regularity and stability. Applicable Anal. 13 (1982), no. 3, 219-233. 
[3] R. Chill, A. Haraux; An optimal estimate for the time singular limit of an abstract wave equation. Funkcial. Ekvac. 47 (2004), no. 2, 277-290.

[4] B. F. Esham, R. J. Weinacht; Hyperbolic-parabolic singular perturbations for scalar nonlinearities. Appl. Anal. 29 (1988), no. 1-2, 19-44.

[5] M. Ghisi, M. GobBino; Global existence and asymptotic behavior for a mildly degenerate dissipative hyperbolic equation of Kirchhoff type. Asymptot. Anal. 40 (2004), no. 1, 25-36.

[6] M. Ghisi, M. GobBino; Global-in-time uniform convergence for linear hyperbolicparabolic singular perturbations. Acta Math. Sin. (Engl. Ser.) 22 (2006), no. 4, 1161-1170.

[7] M. Ghisi, M. GobBino; Hyperbolic-parabolic singular perturbation for mildly degenerate Kirchhoff equations: time-decay estimates. J. Differential Equations 245 (2008), no. 10, 2979-3007.

[8] M. Ghisi, M. GobBino; Hyperbolic-parabolic singular perturbation for mildly degenerate Kirchhoff equations: global-in-time error estimates. To appear on Commun. Pure Appl. Anal.

[9] M. Gobbino; Quasilinear degenerate parabolic equations of Kirchhoff type. Math. Methods Appl. Sci. 22 (1999), no. 5, 375-388.

[10] M. GobBino; Singular perturbation hyperbolic-parabolic for degenerate nonlinear equations of Kirchhoff type. Nonlinear Anal. 44 (2001), no. 3, Ser. A: Theory Methods, 361-374.

[11] H. Hashimoto, T. Yamazaki; Hyperbolic-parabolic singular perturbation for quasilinear equations of Kirchhoff type. J. Differential Equations 237 (2007), no. 2, $491-525$.

[12] J. L. Lions; Perturbations singuliéres dans les problèmes aux limites et en control optimal, Lecture Notes in Mathematics, Vol. 323. Springer-Verlag, Berlin-New York, 1973.

[13] M. NakaO, J. BaE; Global existence and decay to the initial-boundary value problem for the Kirchhoff type quasilinear wave equation with a nonlinear localized dissipation. Adv. Math. Sci. Appl. 13 (2003), no. 1, 165-177.

[14] K. OnO; Global solvability for degenerate Kirchhoff equations with weak dissipation. Math. Japon. 50 (1999), no. 3, 409-413. 
[15] J. WIRTH; Scattering and modified scattering for abstract wave equations with time-dependent dissipation. Adv. Differential Equations 12 (2007), no. 10, 11151133.

[16] Y. YAmADA; On some quasilinear wave equations with dissipative terms. Nagoya Math. J. 87 (1982), 17-39.

[17] T. Yamazaki; Asymptotic behavior for abstract wave equations with decaying dissipation. Adv. Differential Equations 11 (2006), 419-456.

[18] T. YAMAZAKI; Hyperbolic-parabolic singular perturbation for quasilinear equations of Kirchhoff type with weak dissipation. Math. Methods Appl. Sci. In press.

[19] T. YAmAzAKI; Hyperbolic-parabolic singular perturbation for quasilinear equations of Kirchhoff type with weak dissipation of critical power. Preprint. 